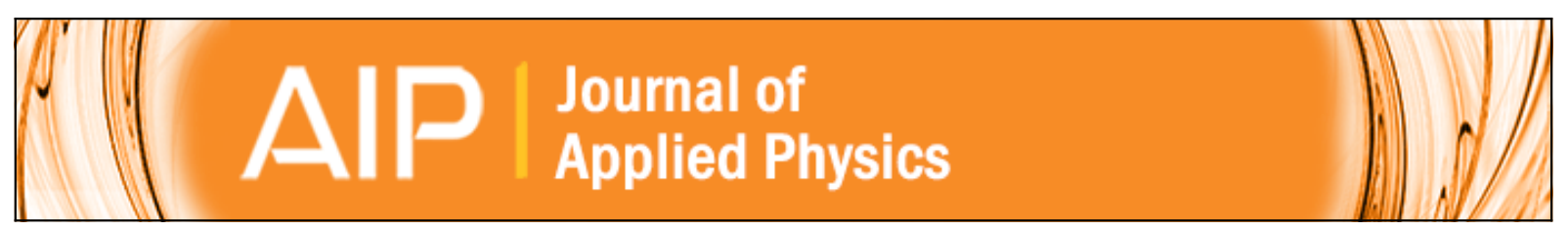

\title{
Photovoltaic phenomena in BiFeO3 multiferroic ceramics
}

C.-M. Hung, C.-S. Tu, W. D. Yen, L. S. Jou, M.-D. Jiang, and V. H. Schmidt

Citation: Journal of Applied Physics 111, $07 D 912$ (2012); doi: 10.1063/1.3675984

View online: http://dx.doi.org/10.1063/1.3675984

View Table of Contents: http://scitation.aip.org/content/aip/journal/jap/111/7?ver=pdfcov

Published by the AIP Publishing

\section{Articles you may be interested in}

Photovoltaic property of domain engineered epitaxial $\mathrm{BiFeO} 3$ films

Appl. Phys. Lett. 105, 252903 (2014); 10.1063/1.4905000

Photovoltaic effect in an indium-tin-oxide/ZnO/BiFeO3/Pt heterostructure

Appl. Phys. Lett. 105, 162903 (2014); 10.1063/1.4899146

Calcium-doping effects on photovoltaic response and structure in multiferroic $\mathrm{BiFeO} 3$ ceramics

J. Appl. Phys. 114, 124105 (2013); 10.1063/1.4823856

High quality multiferroic $\mathrm{BiFeO} 3$ films prepared by pulsed laser deposition on glass substrates at reduced temperatures

J. Appl. Phys. 113, 17D917 (2013); 10.1063/1.4796194

Photo-induced electric phenomena in antiferromagnetic BiFeO3 ceramics

J. Appl. Phys. 113, 17D905 (2013); 10.1063/1.4794743

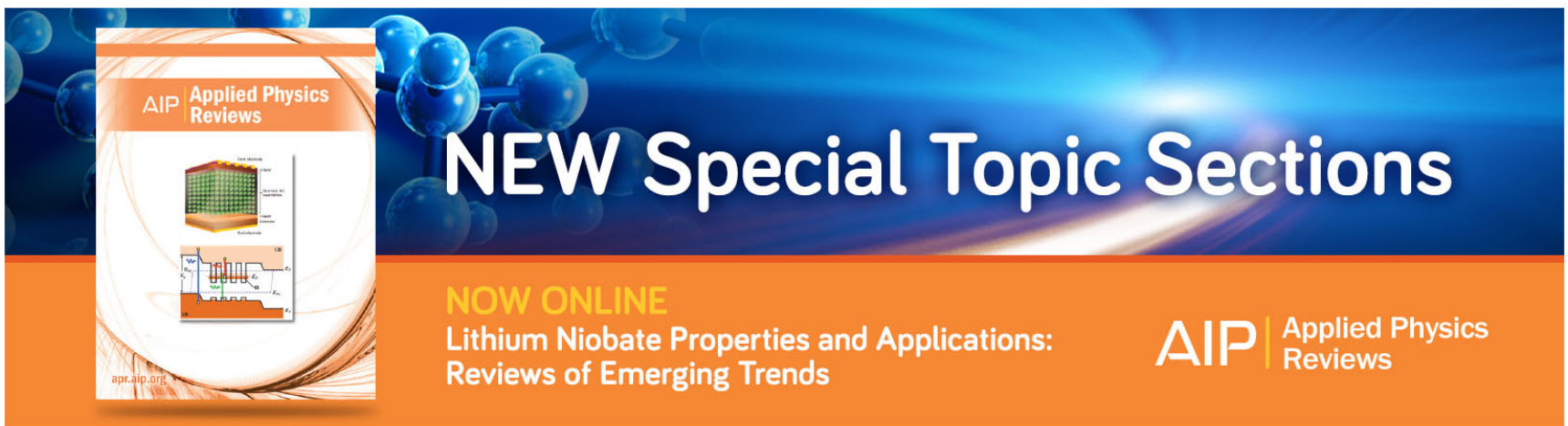




\title{
Photovoltaic phenomena in $\mathrm{BiFeO}_{3}$ multiferroic ceramics
}

\author{
C.-M. Hung, ${ }^{1}$ C.-S. Tu, ${ }^{1,2, a)}$ W. D. Yen, ${ }^{2}$ L. S. Jou, ${ }^{2}$ M.-D. Jiang, ${ }^{2}$ and V. H. Schmidt ${ }^{3}$ \\ ${ }^{1}$ Graduate Institute of Applied Science and Engineering, Fu Jen Catholic University, Taipei 242, Taiwan \\ ${ }^{2}$ Department of Physics, Fu Jen Catholic University, Taipei 242, Taiwan \\ ${ }^{3}$ Department of Physics, Montana State University, Bozeman, Montana 59717, USA
}

(Presented 31 October 2011; received 23 September 2011; accepted 6 November 2011; published online 1 March 2012)

\begin{abstract}
Open-circuit photovoltage and short-circuit photocurrent were investigated in $\mathrm{BiFeO}_{3}(\mathrm{BFO})$ ceramics as functions of laser wavelength $(\lambda=373$ and $532 \mathrm{~nm})$, illumination intensity, and sample thickness. BFO ceramics exhibit significant photovoltaic responses under near-ultraviolet illumination of $\lambda=373 \mathrm{~nm}$. The photovoltaic responses strongly depend on wavelength, light intensity, and sample thickness. The relation between photovoltaic responses and light intensity can be described by exponential equations, $E_{\mathrm{OC}}=E_{\mathrm{S}}[1-\exp (-I / \alpha)]$ and $J_{\mathrm{SC}}=J_{\mathrm{S}}[1-\exp (-I / \beta)] . E_{\mathrm{OC}}, J_{\mathrm{SC}}, E_{\mathrm{S}}$, and $J_{\mathrm{S}}$ are open-circuit photovoltage $(\mathrm{V} / \mathrm{cm})$, short-circuit photocurrent density $\left(\mathrm{A} / \mathrm{cm}^{2}\right)$, saturated photovoltage $(\mathrm{V} / \mathrm{cm})$, and saturated photocurrent density, respectively. This work suggests that $\mathrm{BFO}$ ceramic exhibits stronger photovoltaic responses than the ferroelectric $\mathrm{WO}_{3}$-doped $\mathrm{Pb}_{1-x} \mathrm{La}_{x}\left(\mathrm{Zr}_{y} \mathrm{Ti}_{z}\right)_{1-x / 4} \mathrm{O}_{3}$ ceramics and $\mathrm{Pb}\left(\mathrm{Mg}_{1 / 3} \mathrm{Nb}_{2 / 3}\right)_{1-x} \mathrm{Ti}_{x} \mathrm{O}_{3}$ crystals. C 2012 American Institute of Physics. [doi:10.1063/1.3675984]
\end{abstract}

\section{INTRODUCTION}

Multiferroic materials enable a coupling interaction between ferromagnetic (FM) and ferroelectric (FE) parameters, which attracted much attention in recent years. This magnetoelectric coupling can be utilized to develop additional functionalities that induce magnetization using external electric field or FE polarization using external magnetic field. The multiferroic $\mathrm{BiFeO}_{3}$ (BFO) is perhaps the only material that possesses magnetic and FE properties at and above room temperature. $\mathrm{BFO}$ has a high antiferromagnetic (AFM)-paramagnetic (PM) Néel temperature at $\mathrm{T}_{\mathrm{N}}=350$ $400{ }^{\circ} \mathrm{C}$ and $\mathrm{FE}$ Curie temperature at $\mathrm{T}_{\mathrm{C}}=810-870^{\circ} \mathrm{C}$. ${ }^{1-4}$ The neutron diffraction suggested that $\mathrm{BFO}$ undergoes a first-order transition at $\mathrm{T}_{\mathrm{C}} \cong 820^{\circ} \mathrm{C}$ from a FE rhombohedral to a paraelectric orthorhombic $\mathrm{GdFeO}_{3}$-type structure.,

Photovoltaic effects have been observed in BFO crystal and thin films under illumination of visible light. ${ }^{7-9}$ A large difference in photocurrent between green light $(\lambda=532 \mathrm{~nm})$ and red light $(\lambda=650 \mathrm{~nm})$ indicates that photo-excited carriers across the bulk optical gap $(\sim 2.5 \mathrm{eV})$ dominate the photovoltaic effects. ${ }^{7}$ A strong short-circuit photocurrent was detected in BFO thin films under illumination of $\lambda=460 \mathrm{~nm}$, whose photonic energy is near the bandgap of $2.72 \mathrm{eV} .{ }^{8}$ Strong photovoltaic effects were also observed under near-ultraviolet illumination in FE materials, $\mathrm{WO}_{3}$-doped $\mathrm{Pb}_{1-x} \mathrm{La}_{x}\left(\mathrm{Zr}_{y} \mathrm{Ti}_{z}\right)_{1-x / 4} \mathrm{O}_{3}$ (PLZT) ceramics, and $\mathrm{Pb}\left(\mathrm{Mg}_{1 / 3} \mathrm{Nb}_{2 / 3}\right)_{1-x} \mathrm{Ti}_{x} \mathrm{O}_{3}$ (PMN-PT) crystals. ${ }^{10-13}$ It was found that photo-induced electric response is sensitive to sample thickness and grain size in doped PLZT(3/52/48) ceramics. ${ }^{11,12}$

Though the photovoltaic effects of BFO crystal and thin films have been explored recently for applications of solar cells and optical sensors, a systematic study is still lacking, especially for BFO ceramics. In this work, we have system-

\footnotetext{
a) Author to whom correspondence should be addressed. Electronic mail: 039611@mail.fju.edu.tw.
}

atically investigated the photovoltaic properties of $\mathrm{BFO}$ ceramics and their correlations with light wavelength, illumination intensity, and sample thickness.

\section{EXPERIMENTAL PROCEDURES}

The BFO ceramics were prepared by the solid state reaction method. The starting powders of $\mathrm{Bi}_{2} \mathrm{O}_{3}$ and $\mathrm{Fe}_{2} \mathrm{O}_{3}$ (purity $\geq 99.0 \%$ ) were weighed in 1:1 ratio and then mixed in an agate mortar for more than $24 \mathrm{~h}$. The mixture was dried before calcining at $800^{\circ} \mathrm{C}$. The calcined powder was mixed with polyvinyl acetate as a binder for granulation. The ground mixture was then pressed into $1.0 \mathrm{~cm}$-diameter disks, which were sintered at $840^{\circ} \mathrm{C}$ for $2 \mathrm{~h}$. The densities of sintered BFO ceramics are greater than $90 \%$ of the theoretical values. Very minor second phases were observed by $\mathrm{x}$ ray diffraction.

In photovoltaic measurements, two diode lasers of $\lambda=373$ and $532 \mathrm{~nm}$ were used for photo-excited energies. The experimental set-up is given in Fig. 1. Transparent conductive films of indium tin oxide (ITO) and gold film were deposited on the basal surfaces as electrodes by radiofrequency sputtering. The optical cut-off wavelength of ITO film is near $300 \mathrm{~nm}$. No external dc $E$ field was applied previously and during measurements.

\section{RESULTS AND DISCUSSION}

Figure 2 shows the intensity-dependent open-circuit photovoltage $E_{O C}(\mathrm{mV} / \mathrm{cm})$ under illuminations of nearultraviolet $(\lambda=373 \mathrm{~nm})$ and green $(\lambda=532 \mathrm{~nm})$ lasers for thicknesses of $d=0.47$ and $1.08 \mathrm{~mm}$. The $E_{O C}$ exhibits strong dependences of illumination intensity and sample thickness. Similar intensity and thickness dependences were observed on short-circuit photocurrent density $J_{S C}\left(\mu \mathrm{A} / \mathrm{cm}^{2}\right)$, as shown in Fig. 3. While the laser of $\lambda=373 \mathrm{~nm}$ was turned on, a sharp initial peak begins to appear near $1.04 \mathrm{~mW} / \mathrm{cm}^{2}$ and grows as intensity increases. This photo-induced spike 


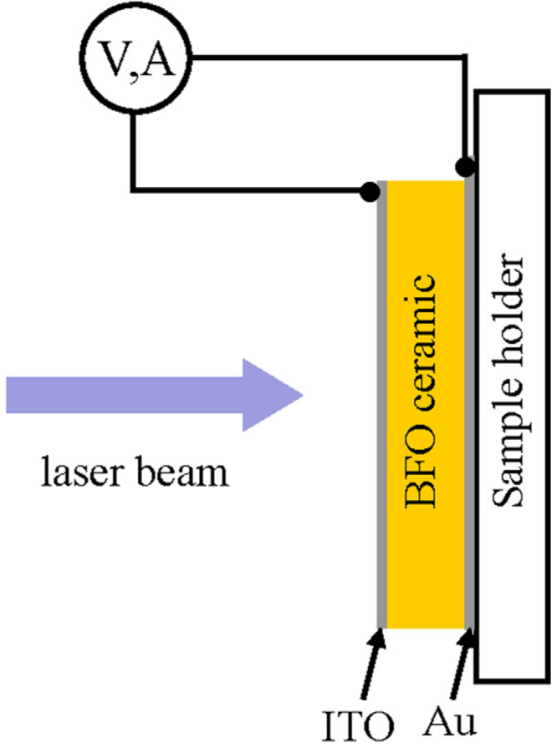

FIG. 1. (Color online) Experimental configuration of photovoltaic measurements.

was likely caused by the pyroelectric effect to the current. ${ }^{14}$ The pyroelectric-effect spike did not occur apparently under illumination of $\lambda=532 \mathrm{~nm}$, due to less absorption of photonic energy.

To understand the correlation between photovoltaic responses and illumination intensity, the averaged photovoltage and photocurrent density were plotted as a function of laser intensity in Figs. 4 and 5 for two thicknesses. As indicated by the solid lines in Figs. 4 and 5, the open-circuit photovoltage $J_{S C}(\mathrm{mV} / \mathrm{cm})$ and short-circuit photocurrent density $E_{O C}\left(\mu \mathrm{A} / \mathrm{cm}^{2}\right)$ can be described as a function of intensity by exponential equations,

$$
E_{O C}=E_{S}\left(1-e^{-I / \alpha}\right) \quad \text { and } \quad J_{S C}=J_{S}\left(1-e^{-I / \beta}\right) .
$$

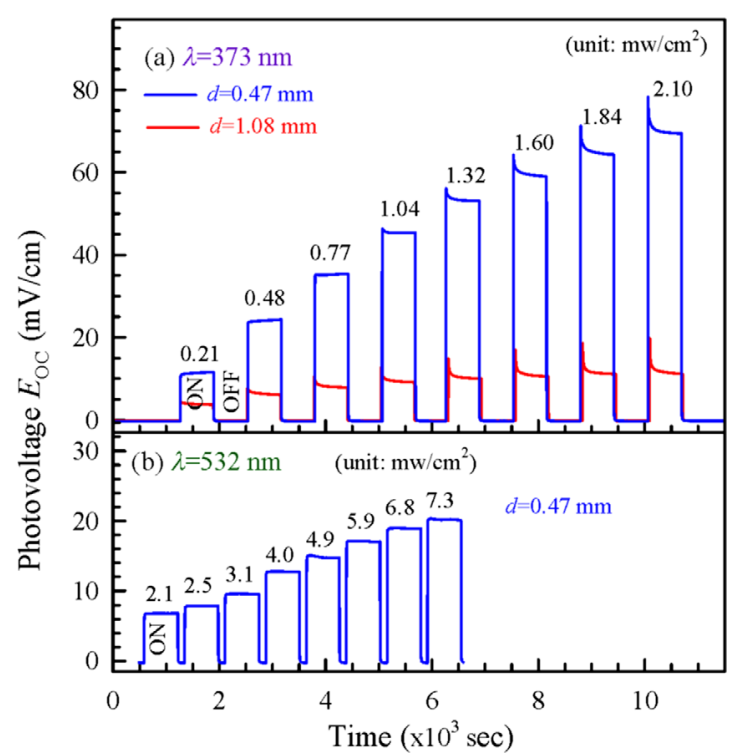

FIG. 2. (Color online) Open-circuit photovoltage varies with time as laser beam is switched on and off with increasing intensity. The intensity $\left(\mathrm{mW} / \mathrm{cm}^{2}\right)$ is labeled on the top of each illumination.

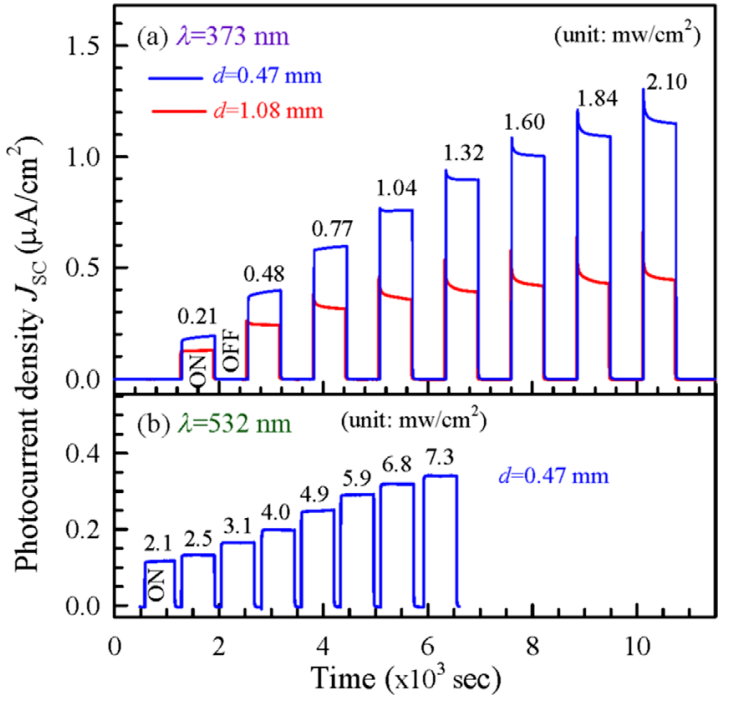

FIG. 3. (Color online) Short-circuit photocurrent density varies with time as laser beam is switched on and off with increasing intensity.

$E_{\mathrm{S}}(\mathrm{mV} / \mathrm{cm}), J_{\mathrm{S}}\left(\mu \mathrm{A} / \mathrm{cm}^{2}\right)$, and $\left(\mathrm{mW} / \mathrm{cm}^{2}\right)$ are saturated open-circuit photovoltage, short-circuit photocurrent density, and illumination intensity, respectively. $\alpha$ and $\beta$ are characteristic constants. The thinner sample has larger $E_{\mathrm{S}}$ and $J_{\mathrm{S}}$, possibly due to fewer barriers in the conducting path. The results show that $\alpha$ and $\beta$ can be the same in photovoltage and photocurrent density for the same thickness. A strongly thickness-dependent photovoltaic behavior was observed in the ferroelectric $\mathrm{WO}_{3}$-doped PLZT ceramics and PMN-PT crystals. ${ }^{10,11,13}$ The short-circuit photocurrent of the poled $\mathrm{WO}_{3}$-doped PLZT (3/52/48) ceramics with thickness $d=50 \mu \mathrm{m}$ exhibits a linear relation with light intensity and is $\sim 20 \mathrm{nA} / \mathrm{cm}^{2}$ at $I \sim 200 \mathrm{~mW} / \mathrm{cm}^{2}$ under illumination of $\lambda=366 \mathrm{~nm} .{ }^{10}$ As shown in Fig. 5, the shortcircuit photocurrent of BFO ceramic with $d=0.47 \mathrm{~mm}$ can reach $\sim 1.2$ and $\sim 0.09 \mu \mathrm{A} / \mathrm{cm}^{2}$ for $I \sim 2.0 \mathrm{~mW} / \mathrm{cm}^{2}$ under illuminations of $\lambda=373$ and $532 \mathrm{~nm}$, respectively.

Compared with $\lambda=532 \mathrm{~nm}$, the near-ultraviolet illumination of $\lambda=373 \mathrm{~nm}$ induces much stronger photovoltaic responses, as shown in Figs. 4 and 5. This indicates that the

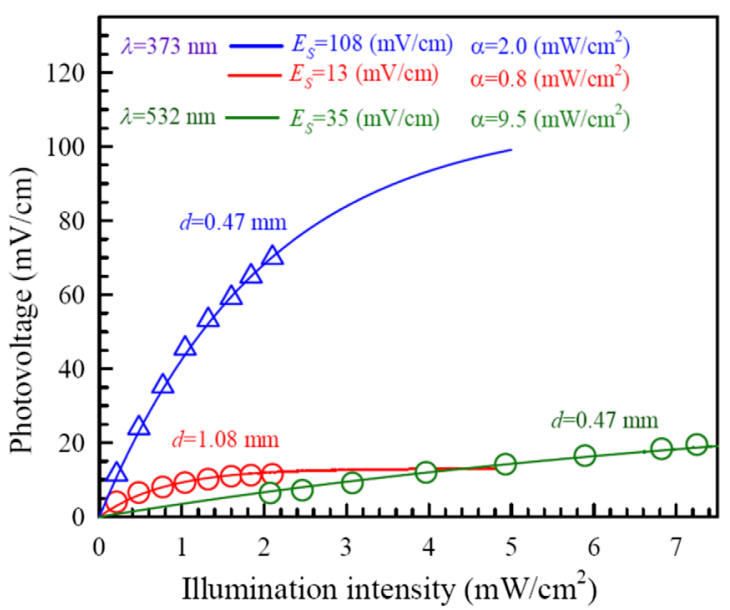

FIG. 4. (Color online) Averaged photovoltage vs intensity. The solid lines are fits of Eq. (1) with parameters given in the figure. 


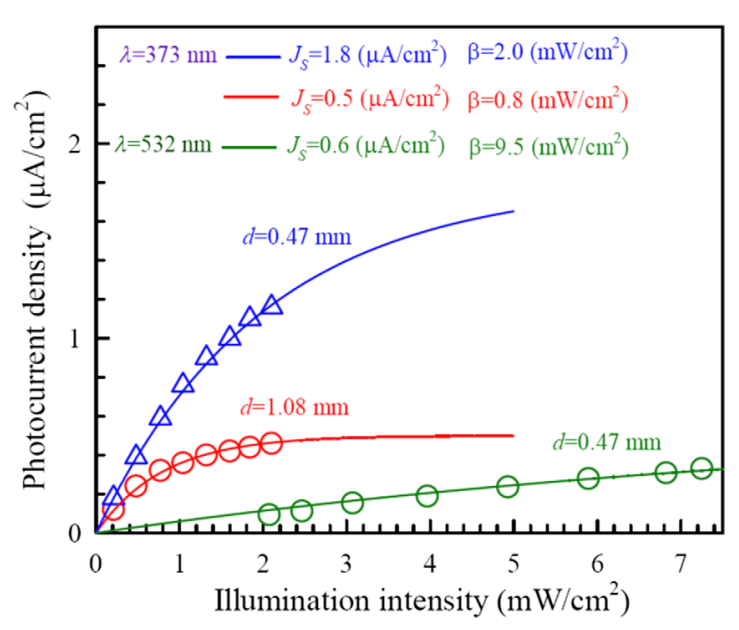

FIG. 5. (Color online) Averaged photocurrent density vs intensity. The solid lines are fits of Eq. (1) with parameters given in the figure.

photonic energy of $\lambda=373 \mathrm{~nm}$ can be absorbed more effectively, because the charge carriers can be excited from the asymmetric potential well through the photonic absorption.

\section{CONCLUSIONS}

The open-circuit photovoltage and short-circuit photocurrent strongly depend on laser wavelength, illumination intensity, and sample thickness. Compared with the green illumination of $\lambda=532 \mathrm{~nm}$, BFO ceramic exhibits a stronger photovoltaic response under near-ultraviolet laser of $\lambda=373 \mathrm{~nm}$. The photovoltage and photocurrent density can be expressed as a function of intensity by exponential equations, $E_{\mathrm{OC}}=E_{\mathrm{S}}[1-\exp (-I / \alpha)]$ and $J_{\mathrm{SC}}=J_{\mathrm{S}}[1-\exp (-I / \beta)]$. Under illumination of $\lambda=373 \mathrm{~nm}$, a sharp pyroelectric response appears while the laser was turned on and increases with laser intensity. The pyroelectric effect does not occur apparently under illumination of $\lambda=532 \mathrm{~nm}$. This work suggests that $\mathrm{BFO}$ ceramics exhibit much stronger photovoltaic responses than the poled $\mathrm{WO}_{3}$-doped PLZT ceramics and high-strain ferroelectric PMN-PT crystals.

\section{ACKNOWLEDGMENTS}

This work was supported by National Science Council of Taiwan Grant No. 100-2112-M-030 -002 -MY3.

${ }^{1}$ J. R. Teague, R. Gerson, and W. J. James, Solid State Commun. 8, 1073 (1970).

${ }^{2}$ P. Fischer, M. Polomska, I. Sosnowska, and M. Szymański, J. Phys. C 13, 1931 (1980).

${ }^{3}$ J.-C. Chen and J.-M. Wu, Appl. Phys. Lett. 91, 182903 (2007).

${ }^{4}$ B. Ramachandran and M. S. Ramachandra Rao, Appl. Phys. Lett. 95, 142505 (2009).

${ }^{5}$ D. C. Arnold, K. S. Knight, F. D. Morrison, and P. Lightfoot, Phys. Rev. Lett. 102, 027602 (2009).

${ }_{6}^{6}$ I. Levin, S. Karimi, V. Provenzano, C. L. Dennis, H. Wu, T. P. Comyn, T. J. Stevenson, R. I. Smith, and I. M. Reaney, Phys. Rev. B 81, 020103 (2010).

${ }^{7}$ T. Choi, S. Lee, Y. J. Choi, V. Kiryukhin, and S.-W. Cheong, Science 324, 63 (2009).

${ }^{8}$ W. Ji, K. Yao, and Y. C. Liang, Adv. Mater. 22, 1763 (2010).

${ }^{9}$ N. A. Spaldin, S.-W. Cheong, and R. Ramesh, Phys. Today 63, 38 (2010).

${ }^{10}$ P. Poosanaas, A. Dogan, S. Thakoor, and K. Uchino, J Appl. Phys. 84, 1508 (1998).

${ }^{11}$ K. Uchino, P. Poosanaas, and K. Tonooka, Ferroelectrics 264, 303 (2001).

${ }^{12}$ K. Takagi, S. Kikuchi, and J.-F. Li, J. Am. Ceram. Soc. 87, 1477 (2004).

${ }^{13}$ C.-S. Tu, F.-T. Wang, R. R. Chien, V. H. Schmidt, T.-M. Hung, and C.-T. Tseng, Appl. Phys. Lett. 88, 032902 (2006).

${ }^{14}$ A. M. Glass, D. von der Linde, and T. J. Negran, Appl. Phys. Lett. 25, 233 (1974). 